\title{
Solving water wave diffraction by an elliptic cylinder using scaled boundary finite element method
}

\author{
L. Tao ${ }^{1} \quad$ H. Song ${ }^{2}$
}

(Received 15 August 2008; revised 19 November 2008)

\begin{abstract}
The scaled boundary finite element method (SBFEM), a novel semianalytical mathematical method, is modified to solve the water wave interaction with an elliptic cylinder. By introducing a virtual circular cylinder surrounding the elliptic cylinder, the whole fluid domain is divided into one unbounded subdomain and several bounded subdomains. The corresponding boundary value problems in bounded and unbounded domains are solved by the SBFEM semi-analytically. Comparisons to the previous numerical solutions demonstrate excellent computational accuracy and efficiency of the present SBFEM approach, as well as the benefit of not suffering from the difficulties of irregular frequency, which are often encountered by the boundary element method. The method can be extended to solve more complex wave structure interaction problems resulting in direct engineering applications.
\end{abstract}

http://anziamj . austms . org. au/ojs/index.php/ANZIAMJ/article/view/1447 gives this article, (c) Austral. Mathematical Soc. 2008. Published December 4, 2008. ISSN 1446-8735. (Print two pages per sheet of paper.) 


\section{Contents}

1 Introduction

C475

2 Theoretical consideration

C477

3 Scaled boundary finite element transformation

C479

4 Solution procedure

C482

4.1 Solution for unbounded subdomain $S_{0} \ldots \ldots \ldots$

C482

4.2 Solution for bounded subdomain . . . . . . . . . . . .

C483

5 Results and discussion

C484

6 Conclusion

C487

References

C488

\section{Introduction}

The interaction of water waves with a vertical cylinder has been studied extensively due to its scientific and engineering significance. However, reports on the studies of the wave diffraction by a cylinder with elliptic cross-section are relatively few. The analytical solution for this problem was first obtained by Chen and Mei [3] via numerical evaluation of Mathieu functions. Considering that the complete analytical solution is too complex and costly for engineering applications, Williams [7] presented approximate methods for calculation of the wave forces and moments on an elliptic cylinder. A number of numerical studies of the wave and elliptic cylinder interaction have been reported using boundary element method (BEM) [1,9] or infinite element method (IEM) [2].

Recently, a new semi-analytical method, namely the scaled boundary 
finite element method (SBFEM) has been successfully applied to the soilstructure interaction problem, combining the advantages of finite element method (FEM) and BEM [8]. The method only discretises the body boundary surface with finite elements, then transforms the governing partial differential equations to ordinary matrix differential equations which are solved analytically. The method solves the problems of singularities more accurately and the problems of unbounded domains more efficiently compared to FEM. Unlike BEM, it does not require a fundamental solution and is free from the irregular frequency difficulty. Fewer elements are needed to obtain very accurate solution.

Only recently has the SBFEM been applied to wave diffraction in which the radiation condition at infinity is required to be satisfied by the scattered waves. Li et al. [4] solved the problem of plane wave diffraction by a vertical cylinder using SBFEM. Similar to the approach of Wolf [8] in obtaining a solution for soil-structure interaction, Li et al. [4] adopted a power series in the form $\left(\sum_{m=0}^{\infty} \mathbf{C}_{m} \bar{\xi}^{-m}\right)$. Since the solution obtained as asymptotic expansions involves sums to infinity, for large values of $\bar{\xi}$ the series approaches the exact solution rapidly and only a few terms in the series need to be computed. However, this is only the case at the cylinder boundary $(\bar{\xi}=$ $k c$, where $k$ and $c$ denote the wave number and the radius of the cylinder respectively) for high frequency waves. For low frequency waves, the series hardly converges to the exact solution.

In this article, water wave diffraction by a vertical elliptic cylinder is solved by SBFEM semi-analytically. By introducing a virtual circular cylinder surrounding the elliptic cylinder, the SBFEM model of Tao et al. [6] is modified and extended to solve a cylinder with elliptic cross section. The fluid domain is divided into an unbounded subdomain and four bounded subdomains. The corresponding boundary value problems in bounded and unbounded domains are all solved by the SBFEM analytically in radial direction. Comparisons to the previous numerical results demonstrate the excellent computation accuracy and efficiency of the present SBFEM approach. Without suffering from 


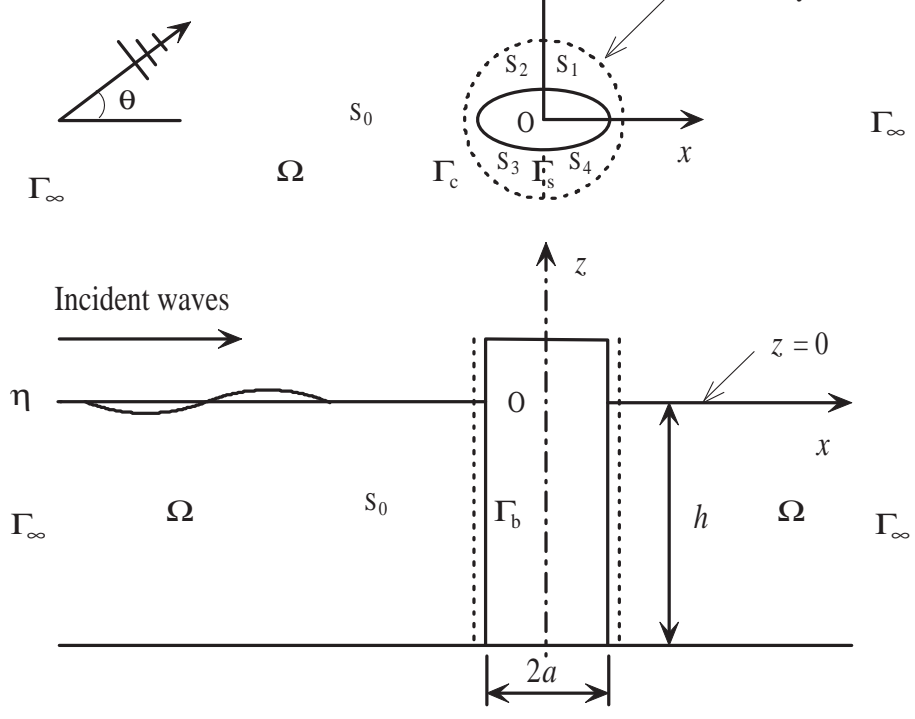

Figure 1: A sketch of the diffraction problem.

the difficulties of irregular frequency and singularity problems, the present model shows additional advantage over BEM.

\section{Theoretical consideration}

Consider a monochromatic plane wave train propagating at an angle $\theta$ with the positive $x$ axis as shown in Figure 1. A fixed vertical elliptic cylinder extends from the sea bottom to the free surface of the ocean along $z$ axis. The origin is placed at the centre of the cylinder on the mean surface level.

Tao et al. [6] showed that the solution process is significantly simplified by choosing the Hankel function as a base function for wave diffraction by 
a circular cylinder. However, it is no longer valid for a cylinder with elliptic cross section. In order to preserve the accuracy and efficiency of the SBFEM model and overcome convergence problems associated with the algebraic series base function, a virtual circular cylinder $\left(\Gamma_{\mathrm{c}}\right)$ surrounding the elliptic cylinder with the same centre is introduced. The whole fluid region is then divided into two regions, the interior region and the region outside of the cylinder $S_{0}$. The interior region is further divided into four subdomains, $S_{1}$, $S_{2}, S_{3}$ and $S_{4}$. The following notation is adopted here: $\Phi_{j}=$ total velocity potential, $\Phi_{0}^{\mathrm{I}}=$ velocity potential of incident wave in $S_{0}, \Phi_{0}^{S}=$ velocity potential of scattered wave in $S_{0}, k=$ wave number, $\omega=$ wave frequency, $\mathrm{h}=$ water depth, $\mathrm{A}=$ amplitude of incident wave, $\mathrm{a}=$ the semiaxis on $x$ axis, $b=$ the semiaxis on $y$ axis, $c=$ the radius of the virtual circular cylinder, $\mathrm{t}=$ time, $\rho=$ mass density of water, and $\mathrm{g}=$ gravitational acceleration. The subscripts $j(j=0,1,2,3,4)$ denote the physical parameters in the solution subdomain $S_{j}$.

Using the water depth function $Z(z)=\cosh k(z+h) / \cosh k h$, to satisfy the seabed boundary conditions, separate the time and spatial dependence as $\Phi_{j}(x, y, z, t)=\phi_{j}(x, y) Z(z) e^{-i \omega t}$. The diffraction problems in $S_{0}$ and $S_{j}$ $(\mathfrak{j}=1,2,3,4)$ are then governed by a Helmholtz equation with the boundary condition at the virtual interface $\Gamma_{\mathrm{c}}$ and the radiation condition at infinity, and the boundary conditions at the interface of the subdomains and body boundary respectively in Table 1 , where $r$ is the radial axis, $i=\sqrt{-1}$ is the imaginary unit, $n$ denotes the normal to the boundary, "adj" in the subscript denotes the physical quantities in the adjacent subdomain, and comma in the subscript designates the partial derivative with respect to the following subscript variable.

The linear incident plane waves are given [5] by the real part of

$$
\Phi_{I}=-\frac{i g A}{\omega} Z(z) e^{i\left(k_{x} x+k_{y} y-\omega t\right)},
$$

and the relationship of the incident, scattered and total velocity potentials 
TABLE 1: The governing equations and boundary conditions.

\begin{tabular}{lll|lll}
\hline \multicolumn{1}{c|}{ unbounded subdomain } & & \multicolumn{3}{c}{ bounded subdomain } \\
\hline$\nabla^{2} \phi_{0}^{S}+k^{2} \phi_{0}^{S}=0$ & in & $S_{0}$ & $\nabla^{2} \phi_{j}+k^{2} \phi_{j}=0$ & in & $S_{j}$ \\
$\phi_{0}^{S}+\phi_{0}^{\mathrm{I}}=\phi_{\text {adj }}$ & on & $\Gamma_{\mathrm{c}}$ & $\phi_{j}=\phi_{\text {adj }}$ & on & $\Gamma_{\mathrm{s}}$ \\
$\phi_{0, \mathrm{n}}^{\mathrm{S}}+\phi_{0, \mathrm{n}}^{\mathrm{I}}=-\phi_{\mathrm{adj}, \mathrm{n}}$ & on & $\Gamma_{\mathrm{c}}$ & $\phi_{j, n}=-\phi_{\text {adj }, n}$ & on & $\Gamma_{\mathrm{s}}$ \\
$\lim _{\mathrm{kr} \rightarrow \infty}(k r)^{1 / 2}\left(\phi_{0, \mathrm{r}}^{\mathrm{S}}-i k \phi_{0}^{S}\right)=0$ & on & $\Gamma_{\infty}$ & $\phi_{j, n}=0$ & on & $\Gamma_{\mathrm{b}}$ \\
\hline
\end{tabular}

are

$$
\Phi_{0}=\Phi_{0}^{\mathrm{I}}+\Phi_{0}^{\mathrm{S}}, \quad \phi_{0}=\phi_{0}^{\mathrm{I}}+\phi_{0}^{\mathrm{S}} .
$$

Table 1 constitutes two sets of the governing equation and boundary conditions for the diffraction of plane waves by an elliptic cylinder with a virtual surrounding circular cylinder, corresponding to boundary value problems in four bounded subdomains and an unbounded subdomain respectively. The boundary condition on the virtual interface is eliminated by matching the unbounded subdomain solution and bounded subdomain solutions on $\Gamma_{\mathrm{c}}$.

\section{$3 \quad$ Scaled boundary finite element transformation}

In this section, $\phi_{j}$ and $\phi_{0}^{S}$ are both denoted as $\phi$ for brevity, and the region $S_{j}$ denoted as $\Omega$. Define the velocity boundary as $\Gamma_{v}$, we have

$$
\phi_{, n}=\bar{v}_{n} \quad \text { on } \quad \Gamma_{v},
$$

where the overbar designates a prescribed value.

SBFEM defines the domain $\Omega$ by scaling a single piecewise smooth curve $S$ relative to a scaling centre $\left(x_{0}, y_{0}\right)$, which is chosen at the cylinder centre in this case, see Figure 2(a). The circumferential coordinate $s$ is anticlockwise 


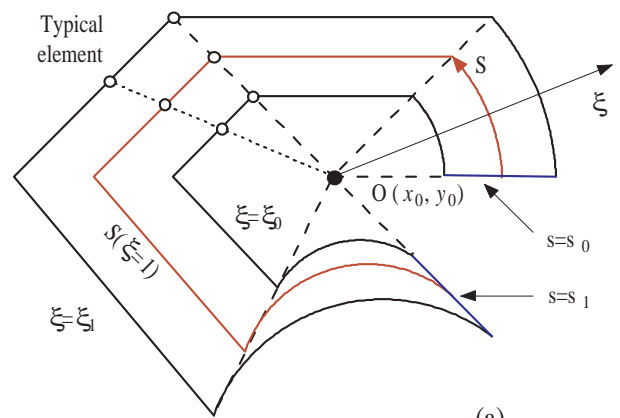

(a)

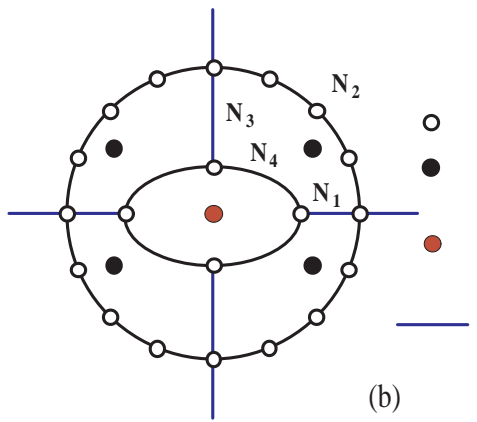

Node

Scaling centre for bounded domain

Scaling centre for unbounded domain

Side face for bounded domain

Figure 2: (a) SBFEM coordinate definition; (b) SBFEM mesh and subdomains.

along the curve $S$ and the normalised radial coordinate $\xi$ is a scaling factor, defined as 1 at curve $S$ and 0 at the scaling centre. The whole solution domain $\Omega$ is in the range of $\xi_{0} \leq \xi \leq \xi_{1}$ and $s_{0} \leq s \leq s_{1}$. The two straight sections $s=s_{0}$ and $s=s_{1}$ are called side faces. They coincide, if the curve $S$ is closed. For bounded domain, $\xi_{0}=0$ and $\xi_{1}=1$; whereas, for unbounded domain, $\xi_{0}=1$ and $\xi_{1}=\infty$. Therefore the Cartesian coordinates are transformed to the scaled boundary coordinate $\xi$ and $s$ with the scaling equations

$$
x=x_{0}+\xi x_{s}(s), \quad y=y_{0}+\xi y_{s}(s) .
$$

By employing SBFEM, an approximate solution of $\phi$ is sought as

$$
\phi_{A}(\xi, s)=\mathbf{N}(s) \mathbf{a}(\xi),
$$

where $\mathbf{N}(s)$ is the shape function and the vector $\mathbf{a}(\xi)$ is analogous to the nodal values same as in FEM. In one element, $\mathbf{N}=\left[\mathrm{N}_{1}, \mathrm{~N}_{2}, \ldots, \mathrm{N}_{\mathrm{p}}\right]$ and $\mathbf{a}=$ $\left[a_{1}, a_{2}, \ldots, a_{p}\right]^{\top}$ where $p$ is the number of nodes in one element. The radial function $a_{\mathfrak{j}}(\xi)$ represents the variation of the wave potential in the radial axis $\xi$ at each node $\boldsymbol{j}$, and $\mathbf{N}(s)$ interpolates between the nodal potential values in the circumferential axis $s$. 
By performing scaled boundary transformation as shown in detail by Tao et al. [6], the following equations are obtained

$$
\begin{gathered}
\mathbf{q}\left(\xi_{1}\right)=\int_{S} \mathbf{N}(s)^{\mathrm{T}}\left(\bar{v}_{n}\left(\xi_{1}, s\right)\right) \xi_{1} d s, \\
\mathbf{q}\left(\xi_{0}\right)=-\int_{S} \mathbf{N}(s)^{\top}\left(\bar{v}_{n}\left(\xi_{0}, s\right)\right) \xi_{0} d s,
\end{gathered}
$$

$\mathbf{E}_{0} \xi^{2} \mathbf{a}(\xi)_{, \xi \xi}+\left(\mathbf{E}_{0}+\mathbf{E}_{1}^{\top}-\mathbf{E}_{1}\right) \xi \mathbf{a}(\xi)_{, \xi}-\mathbf{E}_{2} \mathbf{a}(\xi)+k^{2} \xi^{2} \mathbf{M}_{0} \mathbf{a}(\xi)=\xi \mathbf{F}_{s}(\xi)$,

where $\mathbf{q}(\xi)=\mathbf{E}_{0} \xi \mathbf{a}(\xi)_{, \xi}+\mathbf{E}_{1}^{\top} \mathbf{a}(\xi)$,

$$
\begin{aligned}
& \mathbf{E}_{0}=\int_{S} \mathbf{B}_{1}(s)^{\mathrm{T}} \mathbf{B}_{1}(s)|J| d s, \quad \mathbf{E}_{1}=\int_{\mathrm{S}} \mathbf{B}_{2}(s)^{\mathrm{T}} \mathbf{B}_{1}(s)|J| d s, \\
& \mathbf{E}_{2}=\int_{S} \mathbf{B}_{2}(s)^{\mathrm{T}} \mathbf{B}_{2}(s)|J| d s, \quad \mathbf{M}_{0}=\int_{S} \mathbf{N}(s)^{\mathrm{T}} \mathbf{N}(s)|J| d s, \\
& \mathbf{F}_{s}(\xi)=\mathbf{N}\left(s_{0}\right)^{\mathrm{T}}\left(-\bar{v}_{n}\left(\xi, s_{0}\right)\right)\left|J\left(s_{0}\right)\right|+\mathbf{N}\left(s_{1}\right)^{\mathrm{T}}\left(-\bar{v}_{n}\left(\xi, s_{1}\right)\right)\left|J\left(s_{1}\right)\right|, \\
& \mathbf{B}_{1}(s)=\mathbf{b}_{1}(s) \mathbf{N}(s), \quad \mathbf{B}_{2}(s)=\mathbf{b}_{2}(s) \mathbf{N}(s)_{, s} \\
& \mathbf{b}_{1}(s)=\frac{1}{|J|}\left[\begin{array}{c}
y_{s}(s)_{, s} \\
-x_{s}(s)_{, s}
\end{array}\right], \quad \mathbf{b}_{2}(s)=\frac{1}{|J|}\left[\begin{array}{c}
-y_{s}(s) \\
x_{s}(s)
\end{array}\right],
\end{aligned}
$$

and $|\mathrm{J}|$ is the Jacobian at the boundary

$$
|J|=x_{s}(s) y_{s}(s)_{, s}-y_{s}(s) x_{s}(s)_{, s} .
$$

Equation (8) is the so-called scaled boundary finite element equation with the unknown variable $\mathbf{a}(\xi)$. By introducing the shape function, the Helmholtz equation has been weakened in the circumferential direction, so that the governing partial differential equation is transformed to an ordinary matrix differential equation in radial direction. The rank of matrices $\mathbf{E}_{0}$, $\mathbf{E}_{1}, \mathbf{E}_{2}, \mathbf{M}_{0}$ and vector $\mathbf{a}(\xi)$ is $\boldsymbol{m}$ (where $\boldsymbol{m}$ is the number of nodes in the curve $S$ ). In the present study, the side faces are impermeable so that the flow across the side faces is zero, leading the non-homogeneous term $\mathbf{F}_{s}(\xi)=0$. 
Therefore, the final governing equation (8) is a homogeneous second order ordinary differential equation in terms of a matrix of rank $\mathrm{m}$.

Boundary conditions are weakened in the form of equations (6) and (7), indicating the relationship between the integrated nodal flow on the boundary and the velocity potentials of the nodes. For the wave diffraction problem in the unbounded region, $\xi_{0}=1$ on the boundary of virtual cylinder and $\xi_{1}=+\infty$ at infinity. For the boundary value problem in the bounded region, $\xi_{0}=0$ and $\xi_{1}=1$.

\section{Solution procedure}

\subsection{Solution for unbounded subdomain $S_{0}$}

The solution for unbounded subdomain was obtained by Tao et al. [6] as

$$
\mathbf{a}_{0}(\zeta)=\sum_{j=1}^{m} c_{j} H_{r_{j}}(\zeta) \mathbf{T}_{j}=\mathbf{T H}(\zeta) \mathbf{C},
$$

where $\zeta=k_{c} \xi$ and $r_{j}=\sqrt{\lambda_{j}}, \lambda_{j}$ are the eigenvalues of matrix $\mathbf{E}_{0}^{-1} \mathbf{E}_{2}$, $\mathbf{T}_{j}\left(\mathbf{T}=\left[\mathbf{T}_{1}, \ldots, \mathbf{T}_{m}\right]\right)$ are the eigenvectors of $\mathbf{E}_{0}^{-1} \mathbf{E}_{2}, c_{j}$ are coefficients $\left(\mathbf{C}=\left[\mathrm{c}_{1}, \ldots, \mathrm{c}_{\mathrm{m}}\right]^{\mathrm{T}}\right), \mathrm{H}_{\mathrm{r}_{j}}(\zeta)$ are the Hankel functions of the first kind $(\mathbf{H}(\zeta)=$ $\operatorname{diag}\left[\mathrm{H}_{\mathrm{r}_{1}}(\zeta), \ldots, \mathrm{H}_{\mathrm{r}_{\mathrm{m}}}(\zeta)\right]$, where "diag" denotes a diagonal matrix with the elements in the square brackets on the main diagonal).

The boundary condition on $\Gamma_{\mathrm{c}}$ can be written as

$$
\mathbf{q}_{\mathrm{o}}(k \mathrm{c})=\mathrm{kc}_{\mathrm{c}} \mathbf{E}_{\mathrm{O}} \mathbf{T} \mathbf{H}_{\mathrm{bh}} \mathbf{T}^{-1} \mathbf{a}_{0}(k c)=-\left[\int_{\mathrm{S}} \mathbf{N}(\mathrm{s})^{\mathrm{T}} \mathbf{N}(\mathrm{s}) \mathrm{ds}\right] \overline{\mathbf{v}}_{\mathrm{On}}^{\mathrm{S}},
$$

where $\mathbf{H}_{\mathrm{bh}}=\operatorname{diag}\left[\mathrm{H}_{\mathrm{r}_{1}}(k c)^{\prime} / \mathrm{H}_{\mathrm{r}_{1}}(k c), \ldots, \mathrm{H}_{\mathrm{r}_{\mathrm{m}}}^{\prime}(k c) / H_{r_{\mathrm{m}}}(k c)\right]$, and $\overline{\mathbf{v}}_{\mathrm{On}}^{S}$ is the vector of nodal normal velocity of scattered wave on $\Gamma_{\mathrm{c}}$. 


\subsection{Solution for bounded subdomain}

Define

$$
\mathbf{X}(\xi)=\left[\begin{array}{l}
\mathbf{a}(\xi) \\
\mathbf{q}(\xi)
\end{array}\right]
$$

equation (8) is then written as

$$
\zeta \mathbf{X}(\zeta)_{, \zeta}=-\mathbf{Z X}(\zeta)-\zeta^{2} \mathbf{M X}(\zeta)
$$

where $\zeta=\mathrm{ka} \xi$,

$$
\mathbf{M}=\frac{1}{\mathrm{a}^{2}}\left[\begin{array}{cc}
0 & 0 \\
\mathbf{M}_{0} & 0
\end{array}\right] \quad \text { and } \mathbf{Z}=\left[\begin{array}{cc}
\mathbf{E}_{0}^{-1} \mathbf{E}_{1}^{\top} & -\mathbf{E}_{0}^{-1} \\
-\mathbf{E}_{2}+\mathbf{E}_{1} \mathbf{E}_{0}^{-1} \mathbf{E}_{1}^{\top} & -\mathbf{E}_{1} \mathbf{E}_{0}^{-1}
\end{array}\right] .
$$

According to Wolf [8], the Hamiltonian matrix $\mathbf{Z}$ consists of two groups with opposite sign eigenvalues, $\boldsymbol{\Lambda}_{0}$ and $-\boldsymbol{\Lambda}_{0}$. The real parts of eigenvalues in $\boldsymbol{\Lambda}_{0}$ are all nonnegative. The eigenvalue problem is formulated as

$$
\mathbf{Z} \mathbf{J}=-\mathbf{J} \boldsymbol{\Lambda} .
$$

Similar to Wolf [8] and Li et al. [4], the analytical solution of equation (18) is expressed as

$$
\mathbf{X}(\zeta)=\mathbf{J R}(\zeta) \zeta^{\Lambda} \zeta^{\mathbf{U}} \mathbf{D}
$$

where $\mathbf{U}$ is an upper-triangular matrix with zeros on the diagonal, $\mathbf{D}$ is a coefficient vector, and

$$
\mathbf{R}(\zeta)=\mathbf{I}+\zeta^{2} \mathbf{R}_{1}+\zeta^{4} \mathbf{R}_{2}+\cdots+\zeta^{2 \mathrm{k}} \mathbf{R}_{k}+\cdots
$$

Writing $\mathbf{Y}(\zeta)=\zeta^{\mathbf{U}}$ and $\mathbf{K}(\zeta)=\mathbf{J R}(\zeta)$, and partitioning all the matrices into block matrix with $m \times m$ dimensions and block vector with $m \times 1$ dimensions respectively, equation (21) becomes

$$
\mathbf{X}(\zeta)=\left[\begin{array}{ll}
\mathbf{K}_{11} & \mathbf{K}_{12} \\
\mathbf{K}_{21} & \mathbf{K}_{22}
\end{array}\right]\left[\begin{array}{cc}
\zeta^{\boldsymbol{\Lambda}_{0}} & 0 \\
0 & \zeta^{-\boldsymbol{\Lambda}_{0}}
\end{array}\right]\left[\begin{array}{cc}
\mathbf{Y}_{11} & \mathbf{Y}_{12} \\
0 & \mathbf{Y}_{22}
\end{array}\right]\left[\begin{array}{l}
\mathbf{D}_{1} \\
\mathbf{D}_{2}
\end{array}\right]
$$


The value at $\zeta=0$ should be finite, thus $\mathbf{D}_{2}=0$, and we have

$$
\mathbf{q}(\zeta)=\mathbf{Q}(\zeta) \mathbf{A}^{-1}(\zeta) \mathbf{a}(\zeta)
$$

where $\mathbf{A}(\zeta)=\mathbf{K}_{11}(\zeta) \zeta^{\Lambda_{0}} \mathbf{Y}_{11}(\zeta)$ and $\mathbf{Q}(\zeta)=\mathbf{K}_{21}(\zeta) \zeta^{\Lambda_{0}} \mathbf{Y}_{11}(\zeta)$.

Assembling the matrices in the five subdomains and noting the boundary conditions in Table 1, the whole problem is solved.

\section{$5 \quad$ Results and discussion}

In this article, the interfaces of the subdomains are discretised with three node quadratic elements. As shown in Figure 2(b), $N_{1}, N_{2}, N_{3}$ and $N_{4}$ denote the elements number in one of the interfaces of the subdomains accordingly. If the physical problem is symmetric (for example, incident wave angle $\theta=$ $0, \pi / 2, \pi)$, only half of the subdomains need to be discretised.

Table 2 shows the elements numbers for the convergence test, where $\mathrm{N}_{e}$ and $\mathrm{N}$ denote the number of elements on the elliptic cylinder and on all boundaries respectively. For wave diffraction by a circular cylinder, Figure 3 displays that the wave run-ups converge to the analytical solution [5] very quickly as the number of elements increases. Satisfactory numerical results were obtained when merely four elements for $k a=1$ and six elements for $k a=3$ on the structure boundary were used.

Figure 4 is a comparison of the real parts and the imaginary parts of wave run-ups between the present SBFEM and IEM solutions obtained by Bettess and Bettess [2], where $k a=4, a / b=2, \theta=30^{\circ}$. The IEM solution was obtained by two radial elements and 24 circumferential elements. Figure 4 shows that the results from mesh III with only 12 elements on the circumference by SBFEM agree well with the IEM solution. This clearly demonstrates the high efficiency of SBFEM. 
TABLE 2: The elements numbers used in the convergence test

\begin{tabular}{c|cccc|cc|cc}
\hline Mesh & \multicolumn{3}{|c|}{ elements number } & \multicolumn{2}{c|}{ symmetric } & \multicolumn{2}{c}{ nonsymmetric } \\
& $\mathrm{N}_{1}$ & $\mathrm{~N}_{2}$ & $\mathrm{~N}_{3}$ & $\mathrm{~N}_{4}$ & $\mathrm{~N}_{e}$ & $\mathrm{~N}$ & $\mathrm{~N}_{e}$ & $\mathrm{~N}$ \\
\hline I & 1 & 1 & 1 & 1 & 2 & 7 & 4 & 12 \\
II & 1 & 2 & 1 & 2 & 4 & 11 & 8 & 20 \\
III & 1 & 3 & 1 & 3 & 6 & 15 & 12 & 28 \\
VI & 1 & 4 & 2 & 4 & 8 & 20 & 16 & 38 \\
\hline
\end{tabular}
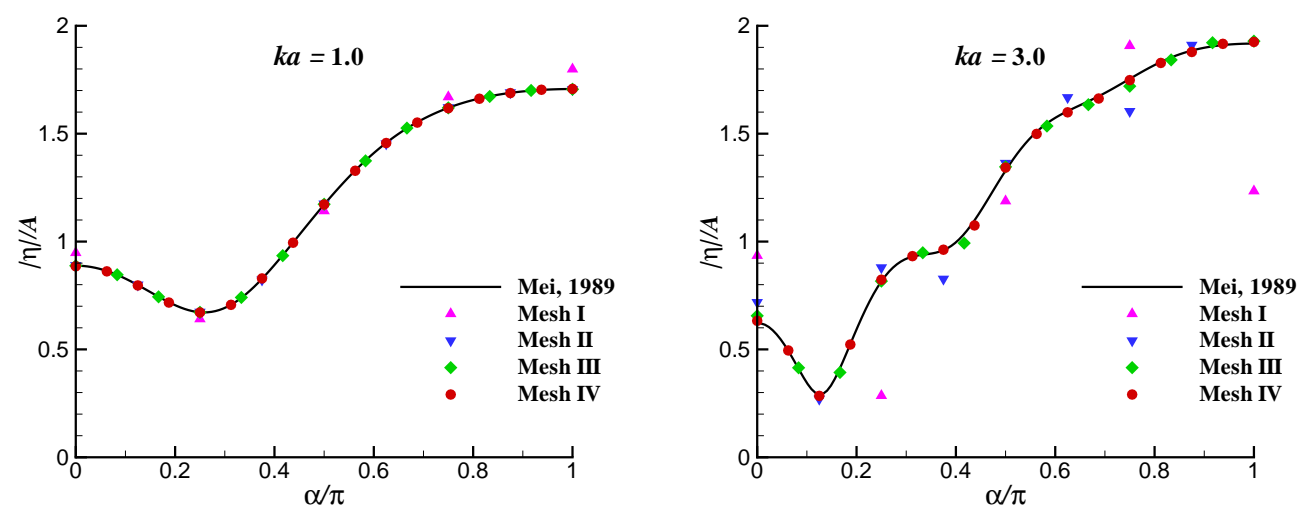

Figure 3: Wave run-ups on a circular cylinder $(\theta=0$ and $\mathrm{c} / \mathrm{a}=1.5)$. 

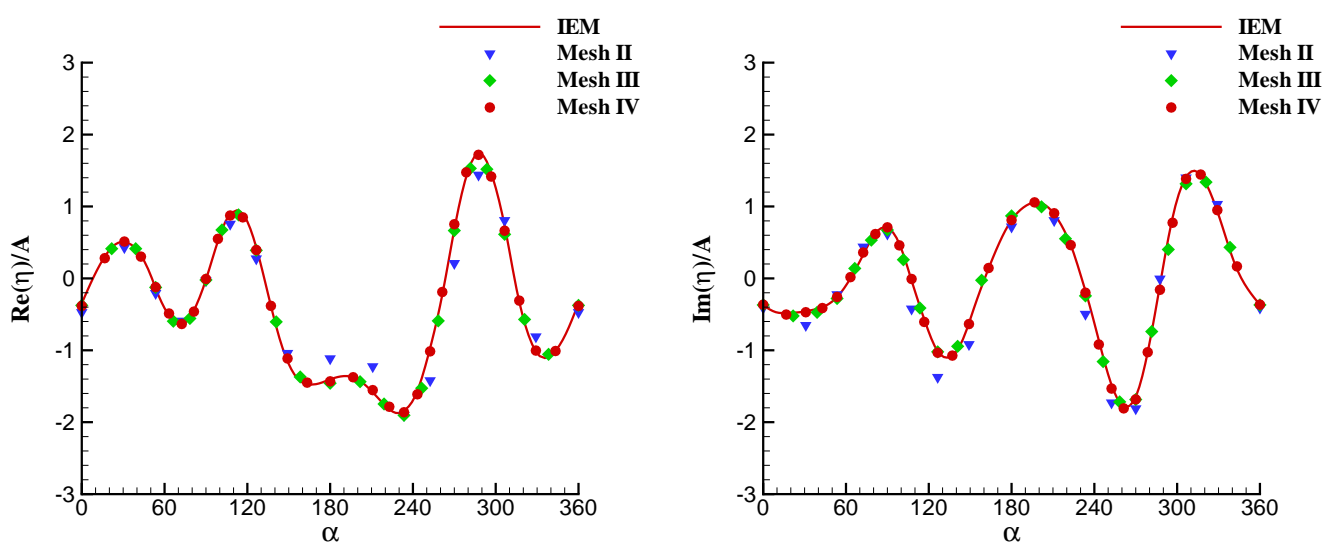

FiguRE 4: Wave run-ups (real and imaginary parts) on the elliptic cylinder.

Replacing the plane wave with a short crested wave as the incident wave in equation (1), the same solution procedure solves short crested wave diffraction by an elliptic cylinder. Figure 5 is a comparison of the short crested wave forces versus incident wave angle $\theta$ between the present SBFEM and BEM solutions, where $k_{x} a=0.8, k_{y} a=0.6, k a=1, a / b=2$. The BEM solution was calculated using 24 constant elements and the almost identical solution is obtained by the SBFEM with even the coarsest mesh, that is, $\mathrm{N}_{1}=\mathrm{N}_{2}=\mathrm{N}_{3}=\mathrm{N}_{4}=1$, a clear demonstration of the high efficiency exhibited in the present SBFEM.

It is noteworthy that the computation times (on a $2.66 \mathrm{GHz}$ Pentium IV PC and MATLAB 7.5) of the SBFEM solutions are very small. For all the cases presented in this article, accurate results are obtained in less than three seconds, a clear demonstration that SBFEM significantly outperforms any current infinite element or boundary element method for similar problems. 

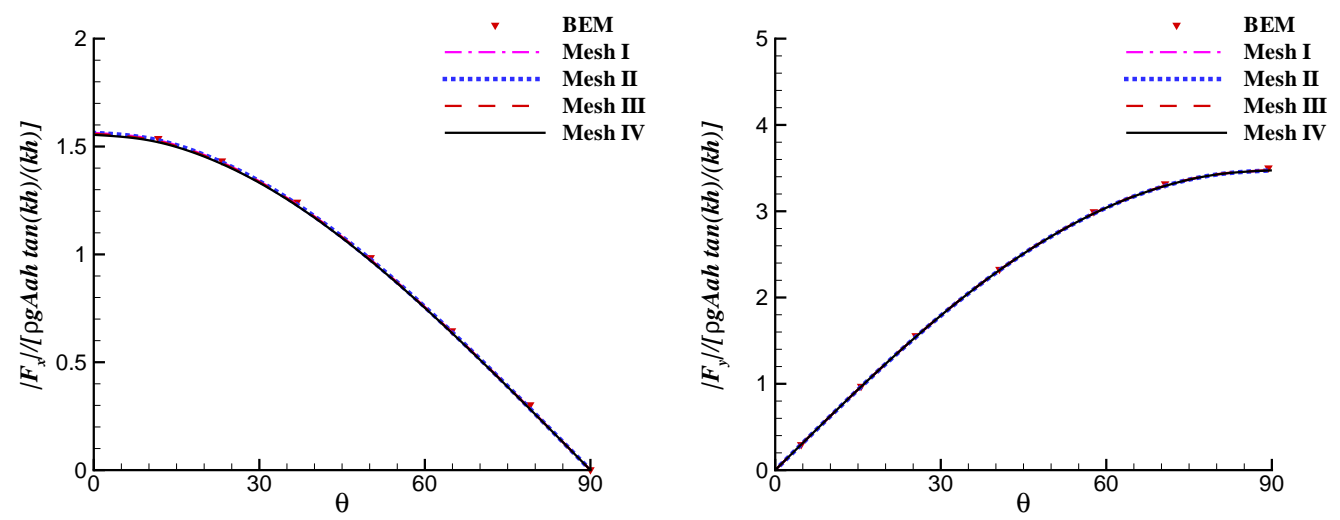

FiguRE 5: Short crested wave forces versus incident wave angle $\theta$.

\section{Conclusion}

In this article, the semi-analytical scaled boundary finite element method is further extended and successfully applied to solve the wave diffraction by a vertical cylinder with an elliptic cross section. Excellent agreements between the present SBFEM solutions and other numerical results are achieved with very low computational cost, demonstrating significant computational accuracy and efficiency - a distinct advantage of the present method for unbounded domain fluid structure interaction problems. This method also has the benefit of not suffering from the difficulties of irregular frequency and singularity, which are often encountered by BEM. The method could be extended to solve more complex wave structure interaction problems with direct engineering applications.

Acknowledgements The first author thanks the financial support from ARC (Grant DP0450906). The second author is grateful for a postdoctoral fellowship at Griffith University to support this research. 


\section{References}

[1] Au, M. C. and Brebbia, C. A., Diffraction of water waves for vertical cylinders using boundary elements, Appl. Math. Model., 7, 1983, 106-114. doi:10.1016/0307-904X(83)90120-8. C475

[2] Bettess, J. A. and Bettess, P., A new mapped infinite wave element for genearal wave diffraction problems and its validation on the ellipse diffraction problem, Comput. Method Appl. M., 164, 1998, 17-48. doi:10.1016/S0045-7825(98)00045-0. C475, C484

[3] Chen, H. S. and Mei, C. C., Wave forces on a stationary platform of elliptical shape, J. Ship Res., 17, 1973, 61-71. C475

[4] Li, B., Cheng, L., Deeks, A. J. and Zhao, M., A semi-analytical solution method for two-dimensional Helmholtz equation, Appl. Ocean Res., 28, 2006, 193-207. doi:10.1016/j.apor.2006.06.003. C476, C483

[5] Mei, C. C., The applied dynamics of ocean surface waves, World Scientific, Singapore, 1989. C478, C484

[6] Tao, L., Song, H. and Chakrabarti, S., Scaled boundary FEm solution of short-crested wave diffraction by a vertical cylinder, Comput. Method Appl. M., 197, 2007, 232-242. doi:10.1016/j.cma.2007.07.025. C476, C477, C481, C482

[7] Williams, A. N., Wave forces on an elliptic cylinder, J. Waterw. Port. C. $A S C E, \mathbf{1 1 1}, 1985,433-449$. http://cedb.asce.org/cgi/WWWdisplay.cgi?8500462. C475

[8] Wolf, J. P., The scaled boundary finite element method, John Wiley and Sons Ltd, Chichester, England, 2003. C476, C483

[9] Zhu, S. and Moule G., Numerical calculation of forces induced by short-crested waves on a vertical cylinder of arbitrary cross-section, Ocean Eng., 21, 1994, 645-662. C475 


\section{Author addresses}

1. L. Tao, Griffith School of Engineering, Griffith University, Queensland 4222, Australia.

mailto:L.Tao@griffith.edu.au

2. H. Song, Griffith School of Engineering, Griffith University, Queensland 4222, Australia. 